\title{
Modeling of charged kaon and neutral kaon fluctuations as a signature for the production of a disoriented chiral condensate in $A-A$ collisions
}

\author{
Ranjit Nayak $\odot,{ }^{1, *}$ Sadhana Dash,,${ }^{1, \dagger}$ Basanta Kumar Nandi,,${ }^{1, \ddagger}$ and Claude Pruneau ${ }^{2, \S}$ \\ ${ }^{1}$ Department of Physics, Indian Institute of Technology Bombay, Mumbai 400076, India \\ ${ }^{2}$ Department of Physics and Astronomy, Wayne State University, Detroit, Michigan 48201,USA
}

(Received 6 August 2019; revised manuscript received 10 February 2020; accepted 13 April 2020; published 13 May 2020)

\begin{abstract}
Anomalous event-by-event fluctuations of the relative yields of neutral $\left(K_{s}^{0}\right)$ and charged kaon $\left(K^{ \pm}\right)$have been predicted to yield a signature for the formation of disoriented chiral condensate (DCC) in relativistic heavy-ion collisions. In this work, we model the production and decay of DCCs in the context of heavy-ion collisions at the CERN Large Hadron Collider, and estimate the sensitivity of large acceptance detectors, such as the ALICE detector, towards the identification of such anomalous decays. Our study is based on the robust statistical observable, $v_{\text {dyn }}$, known for its sensitivity to dynamical fluctuations. We first present simulations without DCCs, based on the HIJING and AMPT models, in order to establish an approximate reference for the magnitude of $v_{\text {dyn }}\left(K^{ \pm}, K_{s}^{0}\right)$ and its centrality evolution in $\mathrm{Pb}-\mathrm{Pb}$ collisions at the $\mathrm{TeV}$ energy scale. We next introduce simple phenomenological models of $K_{s}^{0}$ vs $K^{ \pm}$event-by-event yield fluctuations, which we use to study the feasibility and sensitivity of detection of the production of DCCs in heavy-ion collisions. Although the precision of models such as HIJING and AMPT limit their use as absolute references and thus render anomalous fluctuations difficult to define precisely, our studies demonstrate that the magnitude of $v_{\text {dyn }}\left(K^{ \pm}, K_{s}^{0}\right)$ is in fact very sensitive to the presence of small admixture of DCCs in normal non-DCC events. Consequently, while large values of $v_{\text {dyn }}\left(K^{ \pm}, K_{s}^{0}\right)$ may not be sufficient to identify the existence of DCCs, they nonetheless constitute a first and necessary condition to signal their possible production in heavy-ion collisions.
\end{abstract}

DOI: 10.1103/PhysRevC.101.054904

\section{INTRODUCTION}

Ultrarelativistic heavy-ion collisions at CERN Large Hadron Collider (LHC) energies produce matter consisting of quarks and gluons in a deconfined state. This matter expands and cools through the critical temperature thereby forming hadrons. Within the hot and dense plasma of quarks and gluons, one expects the production of regions where the chiral symmetry is nearly restored. Restoration of chiral symmetry and its subsequent relaxation towards the normal vacuum is additionally posited to yield transient regions where the value of the chiral order parameter differs from that of the surrounding medium. These regions are called disoriented chiral condensate (DCC) [1-3]. Theoretical studies of the production and decay of DCCs were formulated in the context

\footnotetext{
*ranjit@phy.iitb.ac.in

${ }^{\dagger}$ sadhana@phy.iitb.ac.in

‡basanta@phy.iitb.ac.in

§ pruneau@physics.wayne.edu
}

Published by the American Physical Society under the terms of the Creative Commons Attribution 4.0 International license. Further distribution of this work must maintain attribution to the author $(\mathrm{s})$ and the published article's title, journal citation, and DOI. Funded by $S C O A P^{3}$. of the $\mathrm{SU}(2)$ linear $\sigma$ model, more than two decades ago. It was predicted that the production and decay of DCCs shall manifest through enhancement of electromagnetic processes and anomalous distributions of neutral and charged pion multiplicities.

To date, experimental studies of chiral symmetry restoration and searches for the production of DCCs were based on the two-flavor linear $\sigma$ model and involved measurements of pion production and their fluctuations. Unfortunately, these measurements were largely inconclusive [4-7]. More recently, however, observation of a significant enhancement of the production of $\Omega$ (and $\bar{\Omega}$ ) baryons in $17 A \mathrm{GeV}$ in $\mathrm{Pb}-\mathrm{Pb}$ collisions measured at the CERN Super Proton Synchrotron (SPS) provided new insight. It is conceivable that DCC domains also affect the strange particle production. Strange DCC production might then explain the observed enhancement of $\Omega$ observed at the SPS [8]. This implies that the evolution of the chiral condensate could feature a substantial strangeness component. Relaxation to vacuum of the DCC could thus produce kaon isospin fluctuations. One of the first theoretical works to address the observable consequence of kaon fluctuations in the presence of many small DCCs suggested the study of isospin fluctuations in the kaon sector using the $v_{\text {dyn }}$ variable $[9,10]$. This exploration was motivated, in part, by an extension of the two flavor idealization of the linear $\sigma$ model to a three flavor model based on SU(3) symmetry. In this model, fluctuations of strange quarks are coupled to 
those of up and down quarks [11] and the chiral condensate is determined by a $u-d-s$ scalar field. As DCC domains relax, they radiate pions, kaons, and $\eta$ mesons. At low beam energy, the linear $\sigma$ model simulations indicate that pion fluctuations should dominate three flavor DCC behavior given the fraction of energy imparted to kaon fluctuations is very small due to its larger mass. However, this may not be true for collisions at LHC energies where the kaon mass is small relative to the energy available towards particle production. Strangeness might indeed play an important role in the decay of DCCs once the initial system temperature far exceeds the mass of the strange quark. At LHC, a large number of strange/antistrange quarks is rapidly produced during the initial stages of collisions and through the lifetime of the system. Strange quarks are thus available to enter the makeup of DCCs as the system cools down. The rapid and oscillatory relaxation of order parameters would lead to an enhanced production and field fluctuations of kaons, albeit to a lesser degree than pions [9]. DCC production might thus induce anomalous fluctuations of the kaon total isospin measurable in the form of charged vs neutral kaon yields fluctuations. Unfortunately, rather little is known about the dynamics of strange DCC formation compared to that of two quark flavor DCCs. It is also somewhat unclear how the large (on shell) mass of the strange quark might impact the production of DCCs. While such questions are difficult to solve theoretically, experimental evidence for the formation of strange DCCs would likely provide new insights and stimulate renewed interest in DCCs. A search for charged vs neutral kaon yield fluctuations at LHC energies is thus of significant interest.

Experimental observations of DCC signals depend on various factors such as the probability of occurrence of DCC in a collision, the number of DCC domains produced in an event, the size of the domains and the number of particles emitted from these domains, as well as the interaction of these particles with the rest of the collision system [12,13]. Uncertainties about these conditions make the detection of DCC signals quite challenging experimentally. It is thus appropriate to first study kaon yield fluctuations in systems that do not feature DCC production in order to establish a baseline against which measurements of kaon yield fluctuations can be evaluated and compared. The next step is then to introduce a simple model that simulates, qualitatively, the behavior of a system producing kaon DCCs. We shall vary the size and number of such DCCs relative to the full system size.

In this work, we study strangeness isospin fluctuations based on measurements of the relative yield of charged and neutral kaons evaluated with the robust correlator $v_{\text {dyn }}\left(K^{ \pm}, K_{s}^{0}\right)$ [10]. We first establish a baseline for the strength of this correlator with the HIJING [14] and AMPT [15] Monte Carlo event generators. While HIJING does not produce radial and anisotropic flows, known to be important features of heavy-ion collisions, its hadron production involves a cocktail of resonances that may give us a rough estimate of the strength of correlations between charged and neutral kaons. The AMPT model, on the other hand, does produce some radial and anisotropic flow and shall thus give us a first glimpse at the potential impact of such flows on the strength of $v_{\text {dyn }}\left(K^{ \pm}, K_{s}^{0}\right)$. We next formulate a simple phenomenological model of kaon DCC production. Although somewhat simplistic, the model enables us to build intuition as to what minimal size or maximum number of DCCs might be observed in heavy-ion collisions.

Our study is designed to provide a baseline and build our intuition for a measurement of $v_{\text {dyn }}\left(K^{ \pm}, K_{s}^{0}\right)$ carried out by the ALICE collaboration with $\mathrm{Pb}-\mathrm{Pb}$ collisions at $\sqrt{s_{N N}}=$ $2.76 \mathrm{TeV}$ [16]. The definition of $v_{\text {dyn }}\left(K^{ \pm}, K_{s}^{0}\right)$ in the context of this measurement and properties of this observables are briefly discussed in Sec. II. Results of simulations carried out with the HIJING and AMPT models are presented in Sec. III, whereas the phenomenological DCC model and its predictions are discussed in Sec. IV. This work is summarized in Sec. V.

\section{STATISTICAL OBSERVABLE ( $\left.v_{\text {dyn }}\right)$}

Within the context of the SU(3) linear $\sigma$ model, DCC domains relax by radiating pions, kaons, and $\eta$ mesons. The relaxation of these disoriented domains is predicted to produce widely fluctuating neutral pion and kaon yields relative to those of charged pions and kaons in a given fiducial acceptance. These fluctuations may be characterized in terms of a neutral pion fraction $f_{\pi}$ and a neutral kaon fraction $f_{K}$. The neutral pion fraction is defined as $f_{\pi}=N_{\pi^{0}} /\left(N_{\pi^{-}}+\right.$ $N_{\pi^{0}}+N_{\pi^{+}}$), where $N_{\pi^{0}}, N_{\pi^{+}}$, and $N_{\pi^{-}}$represent the yields of neutral, positively charged, and negatively charged pions measured within the fiducial acceptance, respectively. The neutral kaon fraction is defined as $f_{K}=\left(N_{K^{0}}+N_{\bar{K}^{0}}\right) /\left(N_{K^{0}}+\right.$ $N_{\bar{K}^{0}}+N_{K^{+}}+N_{\bar{K}^{-}}$, where $N_{K^{0}}, N_{\bar{K}^{0}}, N_{K^{+}}$, and $N_{K^{-}}$represent the yields of neutral kaon, neutral-anti-kaon, positively charged kaons, and negatively charged kaons measured within the fiducial acceptance, respectively. For any given DCC, the neutral pion fraction $f_{\pi}$ is predicted to fluctuate according to the probability density [1]

$$
P\left(f_{\pi}\right)=\frac{1}{2 \sqrt{f_{\pi}}},
$$

whereas the neutral kaon fraction $f_{K}$ shall be maximally fluctuating with a probability density [11]

$$
P\left(f_{k}\right)=1 \text {. }
$$

It should be noted that these probability densities deviate significantly from those expected from "normal hadronic matter" involving clusters or resonance decays, that yield fluctuations of $f_{\pi}$ and $f_{K}$ determined by multinomial distributions with averages $1 / 3$ and $1 / 2$, respectively. Several experimental difficulties arise, however, towards measurement of these fractions. First, measurements of particle yields are impaired by particle losses determined by the limited detection efficiencies of the experimental device, the reconstruction techniques, and identification protocols used in any given analysis. This leads to binomial sampling and a broadening of the variance of measured multiplicities. Second, the pion and kaon yields may fluctuate irrespective of the production of DCCs and in response to fluctuations of the collision centrality. Third, neutral kaons (antikaons) mix as weak eigenstates in the form $K_{s}^{0}$ and $K_{L}^{0}$. The latter is difficult to observe and count on an event-by-event basis, but the former can be easily identified 
and counted based on the hadronic decay mode $K_{s}^{0} \rightarrow \pi^{+} \pi^{-}$. Event-by-event, half of produced neutral kaons and antikaons shall yield $K_{s}^{0}$. One thus expects $\left\langle N_{K^{0}}\right\rangle=\left\langle N_{\bar{K}^{0}}\right\rangle=\left\langle N_{K_{s}^{0}}\right\rangle$. Given the $K_{L}^{0} \mathrm{~s}$ are not measured ab initio, the $K_{s}^{0}$ yield may thus be regarded as the total neutral kaon yield measured with a $50 \%$ detection efficiency. Evidently, in practice, only those $K_{s}^{0}$ that decay into $\pi^{+} \pi^{-}$will be measured and this observation will have an efficiency of its own. The number of neutral kaons observed on an event-by-event is thus expected to fluctuate, irrespective of the production of DCCs, due to weak mixing and the finite branching ratio of the $K_{s}^{0} \rightarrow$ $\pi^{+} \pi^{-}$decay. Fourth, and last, $K_{s}^{0}$ cannot be observed directly and must be reconstructed and identified statistically by means of topological and invariant mass cuts. Pairs of charged pions of opposite charge may be combinatorially identified as $K_{s}^{0}$ candidates. The number of reconstructed $K_{s}^{0}$ shall then be smeared by the presence of this combinatorial background. Overall, then, it is clear that the fraction $f_{K}$ might fluctuate for a number of reasons having little to do with the existence and production of DCCs. One thus needs a fluctuation observable that is sensitive to the relative yields of produced charged and neutral kaons and robust against losses associated with the production (by mixing) and detection of $K_{s}^{0}$.

Defining the number of measured charged and neutral kaons as $n_{c}=n_{K^{+}}+n_{K^{-}}$and $n_{0}=n_{K_{s}^{0}}$, fluctuations of their relative values may be evaluated with the $v_{\text {dyn }}\left(n_{c}, n_{0}\right)$ observable defined as

$$
v_{\text {dyn }}(\alpha, \beta)=R_{\alpha \alpha}+R_{\beta \beta}-2 R_{\alpha \beta},
$$

where the correlators $R_{\alpha \beta}$ are calculated according to

$$
R_{\alpha \beta}=\frac{\left\langle n_{\alpha}\left(n_{\alpha}-\delta_{\alpha, \beta}\right)\right\rangle}{\left\langle n_{\alpha}\right\rangle\left\langle n_{\beta}\right\rangle}-1
$$

with $\delta_{\alpha, \beta}=1$ for $\alpha=\beta$ and $\delta_{\alpha, \beta}=0$ otherwise. It is straightforward to verify that the correlators $R_{\alpha \beta}$ are robust against experimental efficiencies [10], i.e., they are equal to the values of these correlators obtained based on the true (produced) multiplicities $N_{c}=N_{K^{+}}+N_{K^{-}}$and $N_{0}=N_{K_{s}^{0}}$. Corrections for contamination and admixtures of combinatorial backgrounds are possible by measuring their correlators $R_{\alpha \beta}$ explicitly.

The magnitude of $v_{\mathrm{dyn}}\left(n_{c}, n_{0}\right)$ is determined by the relative strength of charged and neutral kaon correlations: $R_{c c}$ measures the strength of charged kaon correlations, $R_{00}$ measures the strength of neutral kaon correlations, and $R_{c 0}$ is sensitive to charged-neutral kaon correlations. Together, the three terms measure the strength of fluctuations of the difference of the number of charged and neutral kaons $N_{c}-N_{0}$. Thus, $v_{\text {dyn }}\left(n_{c}, n_{0}\right)$ is sensitive to fluctuations of the neutral fraction $f_{K}$. Given it automatically accounts and corrects physical and experimental effects causing particle losses, it then constitutes a practical observable for a measurement of neutral vs charged kaon yield fluctuations.

Note that the individual terms $R_{\alpha \beta}$ shall vanish in the absence of pair correlations, i.e., for Poissonian particle production, and their magnitude is expected to approximately scale in inverse proportion of the total multiplicity of heavyion collisions [10].

\section{HIJING AND AMPT MODEL PREDICTIONS}

We carried out calculations of $v_{\text {dyn }}\left(n_{c}, n_{0}\right)$ for $\mathrm{Pb}-\mathrm{Pb}$ collisions at $\sqrt{s_{N N}}=2.76 \mathrm{TeV}$ simulated with the HIJING and AMPT event generators to establish an approximate baseline for the magnitude of charge vs neutral kaon fluctuations to be expected in the absence of strange DCC production. A total of $3 \times 10^{6}$ HIJING events were generated, whereas $39 \times 10^{6}$, $53 \times 10^{6}$, and $39 \times 10^{6}$ events were produced with AMPT using options (1) string-melting on (SON) and rescattering off (ROFF), (2) string-melting off (SOFF) and rescattering on (RON), and (3) string melting on and rescattering on, respectively. No momentum smearing or particle losses were used in the simulations and $K_{S}^{0}$ were not decayed. Events were partitioned into five centrality classes based on the fractional cross section (number of events) studied as a function of the total charged particle multiplicity observed in the pseudorapidity range $2.8 \leqslant \eta \leqslant 5.1$ and $-3.7 \leqslant \eta \leqslant-1.7$. Events were then analyzed at generator level, in each centrality class, by selecting $K^{ \pm}$and $K_{S}^{0}$ in the transverse momentum range $0.2<p_{\mathrm{T}}<1.5 \mathrm{GeV} / c$ and the pseudorapidity range $|\eta| \leqslant 0.5$ to mimic the conditions of an ongoing ALICE analysis [16]. The number of charged, $N_{c}$, and neutral, $N_{0}$, kaons were counted event-by-event and used to compute event-ensemble averages $\left\langle N_{c}\right\rangle$ and $\left\langle N_{0}\right\rangle$, and second factorial moments $\left\langle N_{c}\left(N_{c}-1\right)\right\rangle,\left\langle N_{0}\left(N_{0}-1\right)\right\rangle$, and $\left\langle N_{c} N_{0}\right\rangle$. In turn, these were combined to compute the correlators $R_{c c}, R_{00}, R_{c 0}$, and $v_{\text {dyn }}\left(N_{c}, N_{0}\right)$ according to Eq. (4), in each centrality class.

The HIJING and AMPT generators do not include DCC production but otherwise feature many of the physical processes required to model heavy-ion collisions. They, thus, provide us a rough baseline set of values of $v_{\text {dyn }}$ to be expected in the absence of DCC formation. We explore in the next section how the production of DCC may then modify these basic expectations. Additionally note that given HIJING does not feature flow-like collectivity, one should expect its predictions of $v_{\text {dyn }}$ to exhibit a simple $1 / N$ scaling with collision centrality. It shall then provide us with a basic reference to study the evolution of $v_{\text {dyn }}$ with collision centrality and possible departure from this scaling associated with the production of DCC in midcentral to central collisions.

Figure 1 shows a graph of $v_{\text {dyn }}$ vs centrality class obtained with HIJING and the three AMPT dynamical modes. In all four cases shown, values of $v_{\text {dyn }}$ exhibit a monotonic increase from $0-10 \%$, corresponding to most central collisions, to $70 \%$, corresponding to the most peripheral collisions studied in this analysis. Additionally, small differences in magnitude are observed, at any given centrality, between the three AMPT dynamical modes considered. However, their trend with collision centrality remain similar. This indicates that $v_{\text {dyn }}$ is indeed sensitive to final state interactions that modify the yield of produced $K^{ \pm}$and $K_{S}^{0}$ as well as their correlations in $\mathrm{Pb}-\mathrm{Pb}$ collisions, even if these effects are small.

In order to account for the dilution of the $R_{2}$ correlators with increasing particle multiplicity (and number of sources), we scale values of $v_{\text {dyn }}$, in Fig. 1(b), by the geometric mean, $\sqrt{\left\langle N_{\mathrm{K}^{ \pm}}\right\rangle\left\langle N_{\mathrm{K}^{0}}\right\rangle}$, of the numbers of $K^{ \pm}$and $K_{S}^{0}$ produced in the fiducial acceptance of the measurement. As expected, scaled values calculated with HIJING and AMPT/RON are 


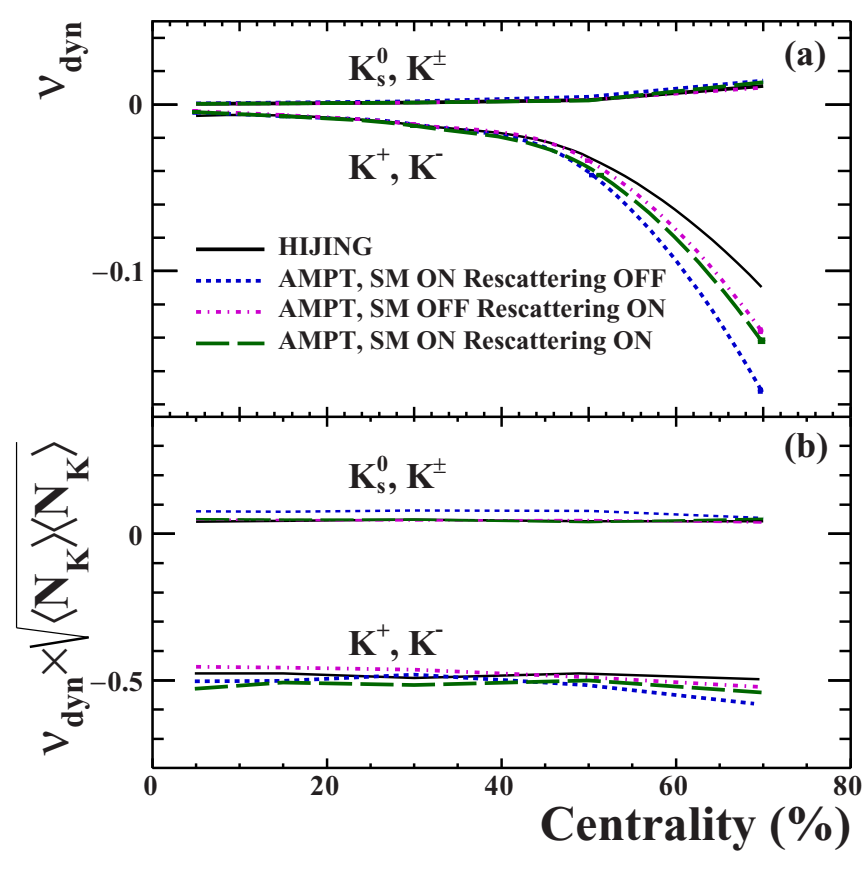

FIG. 1. (a) HIJING and AMPT model predictions for the collision centrality dependence of $v_{\mathrm{dyn}}\left(K^{ \pm}, K^{0}\right)$ and $v_{\mathrm{dyn}}\left(K^{+}, K^{-}\right)$in $\mathrm{Pb}-\mathrm{Pb}$ collisions at $\sqrt{s_{N N}}=2.76 \mathrm{TeV}$. (b) $v_{\text {dyn }}$ values scaled by the geometric mean of kaon multiplicities.

essentially invariant with collision centrality, whereas scaled values calculated with $\mathrm{AMPT} / \mathrm{SON} / \mathrm{ROFF}$ exhibit a weak collision centrality dependence, owing to the production of higher mass states in most central collisions. Overall, both HIJING and AMPT produce nearly invariant values of $v_{\mathrm{dyn}} \sqrt{\left\langle N_{\mathrm{K}^{ \pm}}\right\rangle\left\langle N_{\mathrm{K}^{0}}\right\rangle}$ showing that the expected $1 / N$ dilution is approximately verified.

It is interesting to compare calculations of $v_{\text {dyn }}\left(N_{c}, N_{0}\right)$, discussed above, with predictions for $v_{\mathrm{dyn}}\left(N_{+}, N_{-}\right)$computed with the same models and also shown in Fig. 1(a). Conservation laws, namely electric charge and strangeness conservation, restrict the level of fluctuations possible in the production of charged kaons. The cumulant $R_{+-}$is larger than either of $R_{++}$or $R_{--}$and $v_{\text {dyn }}\left(N_{+}, N_{-}\right)$is consequently negative at all collision centralities. By contrast, charge conservation does not limit the fluctuations of the relative yields of $K^{ \pm}$and $K^{0}$ and $v_{\text {dyn }}\left(N_{c}, N_{0}\right)$ takes positive values at all centralities. Note, however, that the magnitude of $v_{\text {dyn }}\left(N_{+}, N_{-}\right)$influences in part the value of $v_{\mathrm{dyn}}\left(N_{c}, N_{0}\right)$, given

$$
\begin{aligned}
R_{c c} & =f^{2} R_{++}+(1-f)^{2} R_{--}+2 f(1-f) R_{+-} \\
& =\frac{1}{4}\left[2 R_{++}+2 R_{--}-v_{\mathrm{dyn}}\left(N_{+}, N_{-}\right)\right],
\end{aligned}
$$

where $f=\left\langle N_{+}\right\rangle /\left(\left\langle N_{+}\right\rangle+\left\langle N_{-}\right\rangle\right)$and in the second line, we assumed $f=0.5$, which is approximately valid at LHC energy. Finally, note that values of $v_{\mathrm{dyn}}\left(K^{+}, K^{-}\right)$scaled by the number of charged kaons are also invariant with collision centrality, while AMPT/SON/ROFF displays a modest collision centrality dependence.

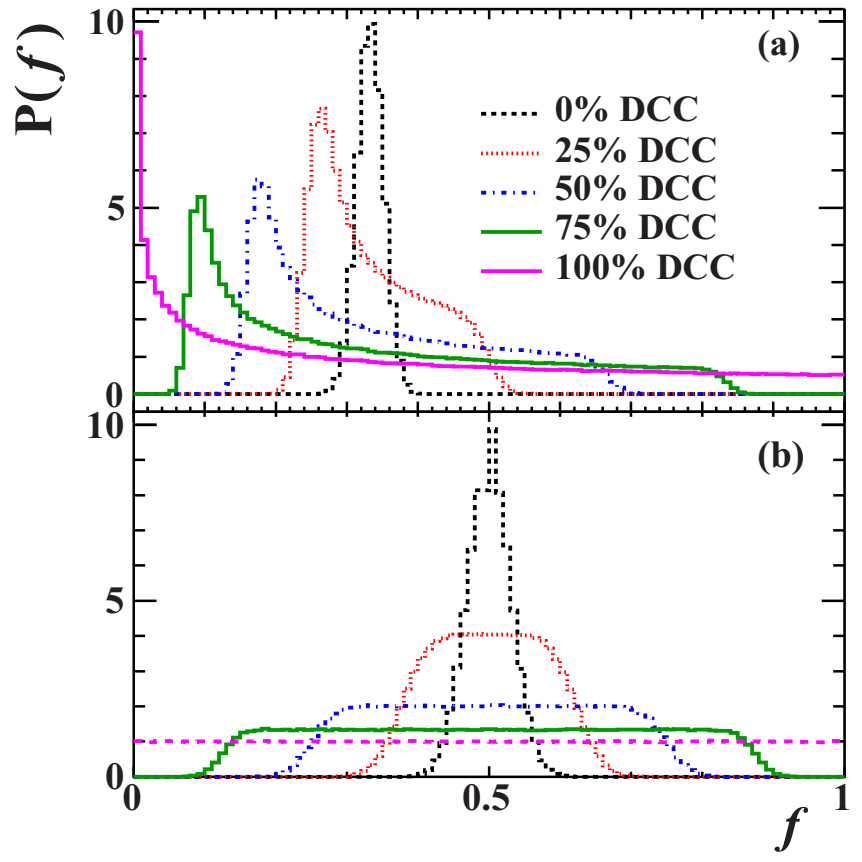

FIG. 2. Probability densities of the fractions of (a) neutral pions and (b) neutral kaons for selected values of the fraction $f_{\mathrm{DCC}}$ of particles produced within DCCs.

\section{DCC MODEL SIMULATIONS}

We simulate the production of DCCs with a simple phenomenological model to gain insight into the correlation strength measurable with $v_{\text {dyn }}$ in the presence of condensates decaying into neutral and charged kaons. The DCC simulator is designed to match the pion and kaon multiplicity production observed experimentally and involves "normal" and DCC particle generation components. The former produces charged and neutral particles based on a binomial distribution, whereas the DCC particle production is determined by Eqs. (1), (2) for pions and kaons, respectively. Events can be set to consist of DCC matter entirely or a mix of normal and DCC matter by used of a parameter $f_{\mathrm{DCC}}$ which controls the number of particles produced within the binomial and DCC components. The average fraction of particles consisting of kaons is determined by a user selected parameter $f_{K}$ set at simulation startup. However, the kaon to pion yield ratio is allowed to fluctuate according to a binomial distribution determined by this fraction and the total multiplicity. This multiplicity is randomly chosen according to a PDF that approximately replicates the charged particle multiplicity reported by the ALICE collaboration in 0-10\% collision centrality, scaled by a factor of $3 / 2$ to account for neutral particle production. The number of $K_{S}^{0} \mathrm{~s}$ in the DCC and normal parts of an event are randomly generated according to a binomial distribution with a mean probability of 0.5 based on the number of neutral kaons in either parts.

We explore the impact of DCC production by varying the size and number of DCC in generated events. Figure 2 displays the fractions of neutral pions (top panel) and kaons (bottom panel) obtained in generated events with selected 


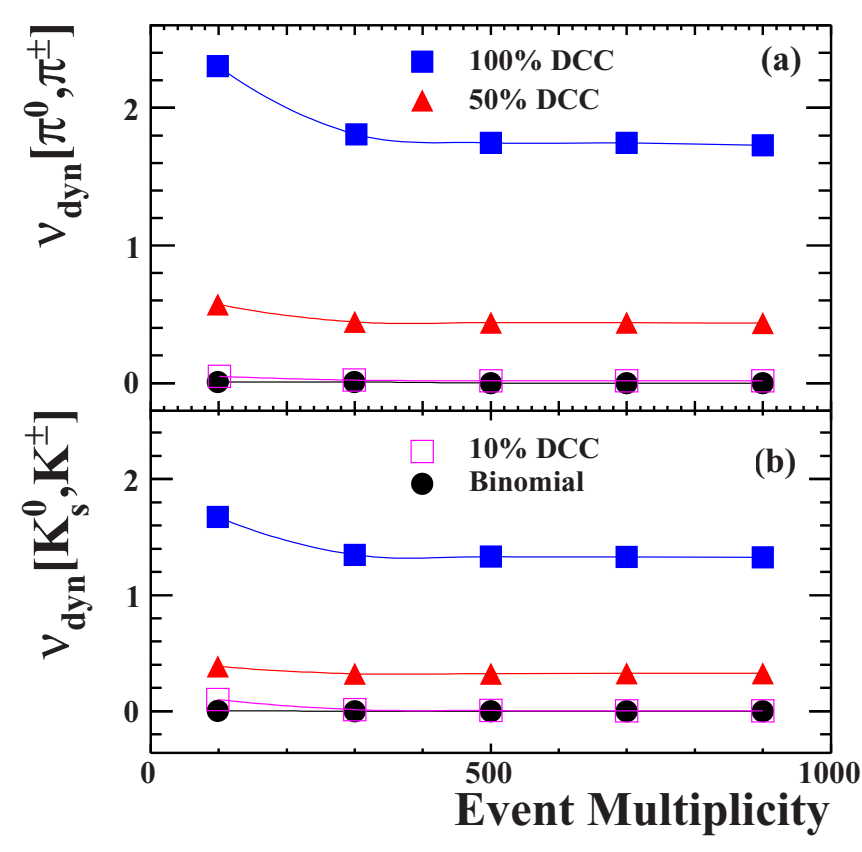

FIG. 3. Evolution of (a) $v_{\text {dyn }}\left(\pi^{0}, \pi^{ \pm}\right)$and (b) $v_{\text {dyn }}\left(K^{0}, K^{ \pm}\right)$, with event multiplicity for selected values of the fraction of particles produced within DCCs.

values of the DCC fraction $f_{\mathrm{DCC}}$. We consider few distinct particle production scenarios: Scenario 1: All generated events are assumed to contain one DCC domain but the size of the domains is varied by changing the fraction of (a) pions and (b) kaons they produce relative to the full system. As shown in Fig. 3, v dyn increases monotonically with the DCC size and reaches very large magnitudes for events containing large DCC domains. The rise of $v_{\mathrm{dyn}}$ at small event multiplicity is due to a decline of the cross-term $R_{c 0}$ relative to $R_{00}$ and $R_{c c}$. Scenario 2: The DCC size is kept fixed and accounts for 100 percent of the event size but the probability of occurrence of DCCs is varied. The magnitude of $v_{\mathrm{dyn}}$ rises with the fraction of events containing a DCC, called $p_{\mathrm{DCC}}$, as illustrated in Fig. 4. Sizeable $v_{\text {dyn }}$ magnitudes and deviations from binomial expectation values occur for probabilities as small as $p_{\text {DCC }}=$ 0.01. Scenario 3: We use HIJING events as a baseline for the description of pion and kaon production and their eventby-event fluctuations. DCC-like fluctuations are introduced by randomizing the charge of kaons produced by HIJING. Figure 5 displays the collision centrality evolution of $v_{\text {dyn }}$ obtained when progressively increasing the fraction of events containing a single (large) strange DCC. As already discussed in Sec. III, the magnitude $v_{\mathrm{dyn}}$ exhibits an approximate $1 / N$ behavior with increasing event multiplicity $N$, shown as a purple line in Fig. 5. Injection of DCC-like fluctuations, however, drastically changes this behavior and the distribution of $v_{\text {dyn }}$ with collision centrality becomes progressively flat (nearly invariant) when rising the fraction of events containing DCClike fluctuations. Values of $v_{\mathrm{dyn}}$ scaled by the geometric mean of the number of kaons, shown in Fig. 5(b), and the charged particle density, shown in Fig. 5(c), are no longer invariant with collision centrality and sharply rise in central $\mathrm{Pb}-\mathrm{Pb}$ collisions. Note that significant deviations from the HIJING

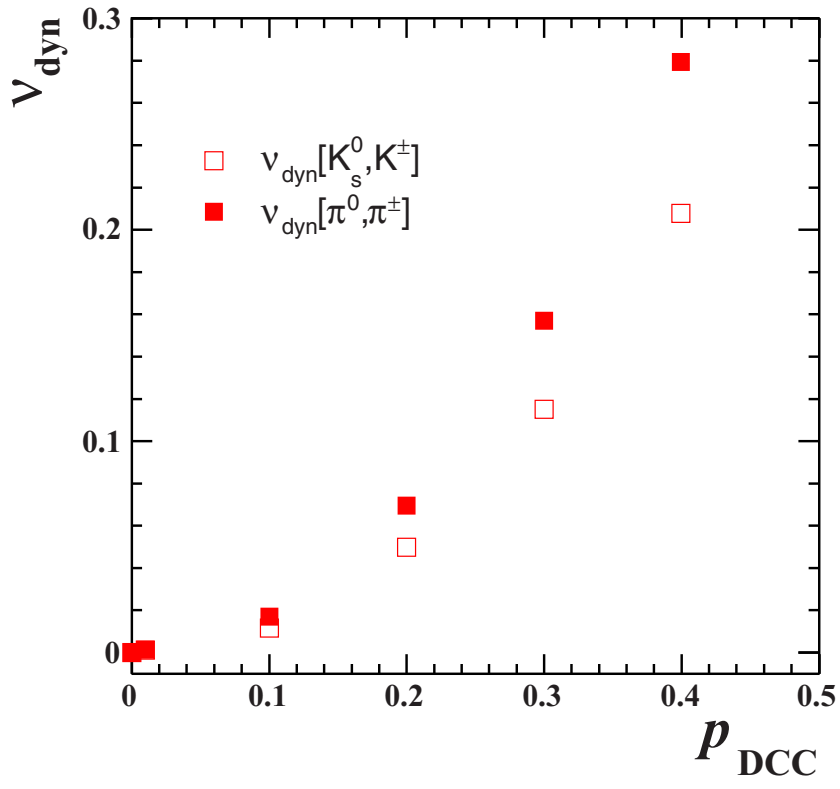

FIG. 4. $v_{\text {dyn }}$ as a function of the fraction of DCC-like events $\left(p_{\mathrm{DCC}}\right)$ for a given multiplicity bin.

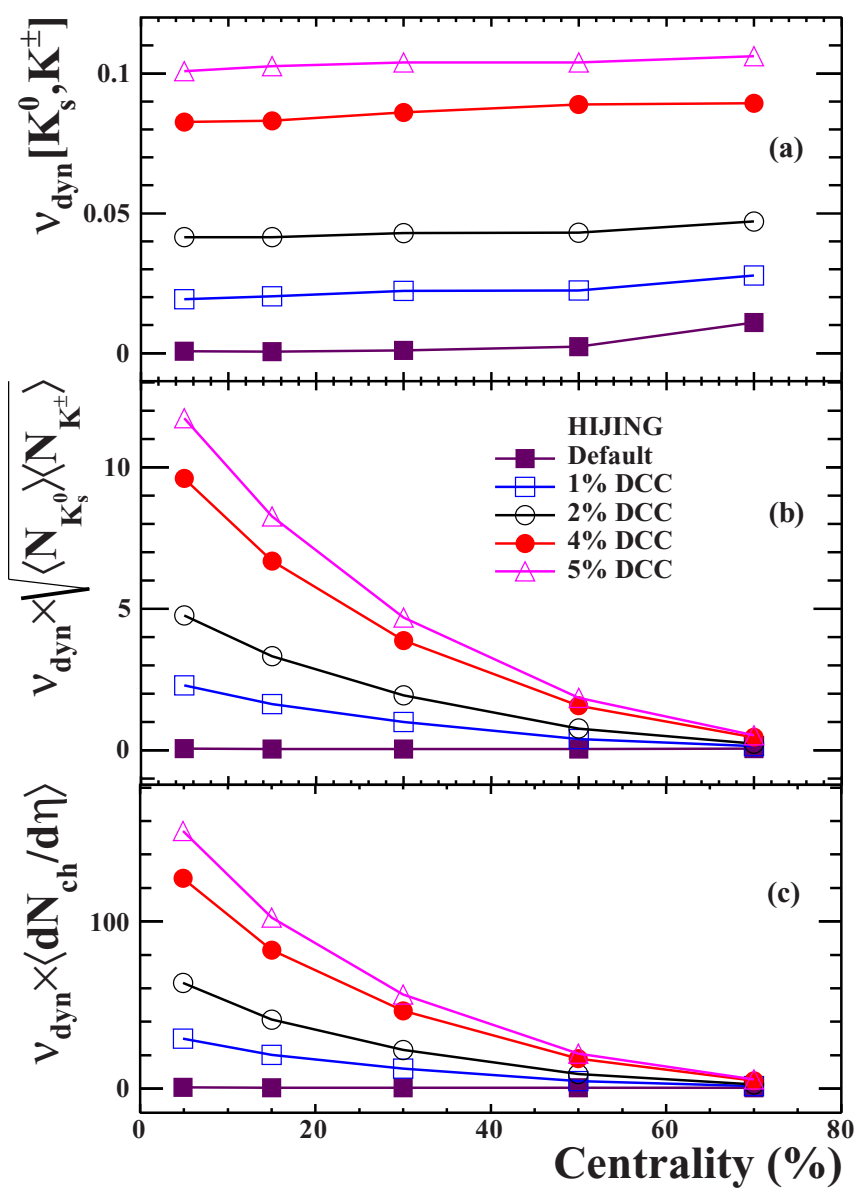

FIG. 5. (a) $v_{\text {dyn }}\left(K_{s}^{0}, K^{ \pm}\right)$vs centrality of $\mathrm{Pb}-\mathrm{Pb}$ collisions at $\sqrt{s_{N N}}=2.76 \mathrm{TeV}$, (b) $v_{\text {dyn }}$ scaled by the kaon multiplicity, (c) $v_{\text {dyn }}$ scaled by the charged particle multiplicity. 


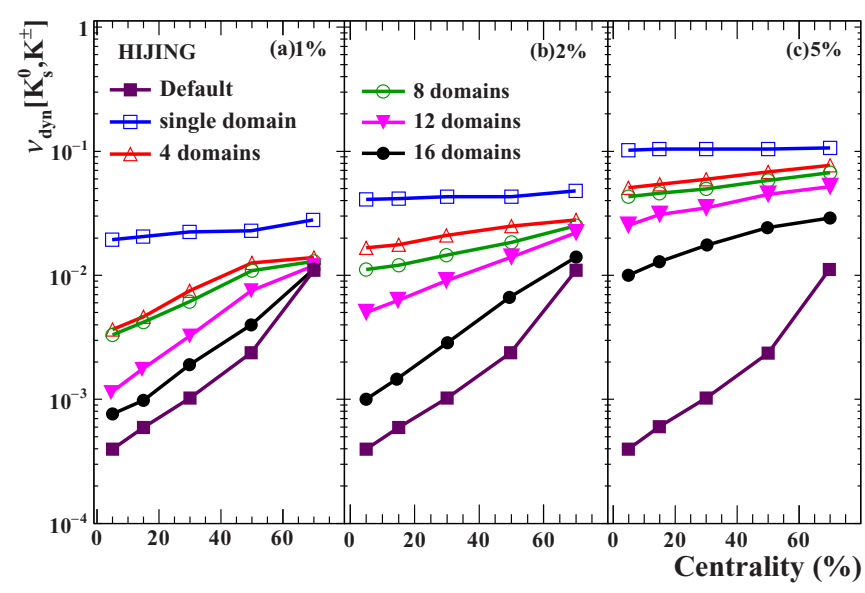

FIG. 6. $v_{\text {dyn }}\left(K_{s}^{0}, K^{ \pm}\right)$vs centrality of $\sqrt{s_{N N}}=2.76 \mathrm{TeV} \mathrm{Pb}-\mathrm{Pb}$ collision involving single and multiple DCC domains per event for DCC fractions of (a) $1 \%$, (b) $2 \%$, and (c) $5 \%$.

baseline are found already when the fraction of DCC amounts to only one percent. The magnitude and collision centrality evolution of $v_{\mathrm{dyn}}$ are thus indeed quite sensitive to the presence of DCC-like fluctuations in $\mathrm{Pb}-\mathrm{Pb}$ collisions. Scenario 4: Generated HIJING events are modified to simulate the production of one or more DCC domains per event by considering different regions in pseudorapidity $\eta$ and azimuth $\phi$. The size of the multiple domains is varied by changing the fraction of kaons on an event-by-event basis. The values of the $v_{\text {dyn }}$ are calculated for events with multiple domains of DCC and compared with the events containing a single DCC domain, obtained for $1 \%, 2 \%$, and 5\% DCC fraction as depicted in Figs. 6(a)-6(c), respectively. We find that with the increase in the number of DCC domains, $v_{d y n}$ progressively approaches the values of the HIJING baseline but remains nonetheless distinct. Indeed, although the production of multiple domains reduces the value of $v_{d y n}$ relative to that observed for a single domain, a centrality dependence distinct from that of default HIJING events containing no DCCs is observed for events with four or more domains. Similar trends are observed for higher values of DCC fractions depicted in Figs. 6(b) and 6(c). It is important to acknowledge that scenario 4 is likely too simplistic given its does not explicitly involve the production of $\Omega$ baryons from strange DCCs as a component of their relaxation mechanism [8]. Additionally, Eqs. (1) and (2) refer to a distribution of particle fractions from the decay of a single DCC domain, which we applied without modifications to cases of multidomains production. If baryon formation is understood in the Skyrmion picture, then neighboring DCC domains could get locked up in creating (topological) winding for the Skyrmion. These neighboring DCC domains would not simply relax and give rise to conventional DCC distributions.
The magnitude of deviations of $v_{d y n}$ from default HIJING values may thus be quantitatively biases. However, properly addressing such complexities in a simulation of the production and decay of strange DCCs is beyond the scope of this work. And although it is difficult to asses the practical impact of DCC neighbor locking and correlations, it is nonetheless reasonably safe to conclude, based on simulations with scenario 4 , that the production of single or multiple DCC domains per event should have a measurable impact on measured values of $v_{\mathrm{dyn}}$ even though the production of DCCs might be a rare occurrence.

\section{SUMMARY}

The event-by-event fluctuations of neutral and charged kaons were studied using the $v_{\text {dyn }}$ observable in Monte Carlo models (HIJING and AMPT) in Pb-Pb collisions at $\sqrt{s_{N N}}=$ $2.76 \mathrm{TeV}$ for various collision centralities. The estimates from these models are relevant to the ongoing experimental search of DCC-like signals in heavy-ion collisions at LHC and BNL Relativistic Heavy Ion Collider (RHIC) as these models do not include the dynamics of DCC physics. A simple phenomenological model was developed to implement the DCC-type events and study the sensitivity of the $v_{\text {dyn }}$ observable to the fraction of DCC events as well as the size of DCC domains. The value of $v_{\mathrm{dyn}}$ increases with a rising fraction of DCClike events in the sample for a given multiplicity class. The variation of $v_{\text {dyn }}$ was also studied as a function of multiplicity for different size of DCC domains. Both studies indicate that $v_{\text {dyn }}$ is very sensitive to the presence of DCC-like events in heavy-ion collisions. Finally, we showed, with simulations based on the HIJING model, that the magnitude of $v_{\mathrm{dyn}}$ is rather sensitive to the presence of a small admixture of DCCs to "normal" collisions. Although $v_{\text {dyn }}$ values predicted by HIJING for normal collisions may not be entirely accurate because this model does not capture all the relevant dynamics of heavy-ion collisions, it is nonetheless clear that even a small admixture of DCCs could lead to a sizable departure from the $1 / N$ scaling and as such constitute an indication, although not a proof, of abnormal and perhaps DCC-like production in heavy-ion collision. Further work is however required to improve predictions of $v_{\mathrm{dyn}}$ based on "normal" particle production scenarios as well as our assessment of deviations, from this normal behavior, caused by small admixture of strange DCCs.

\section{ACKNOWLEDGMENTS}

This work was supported in part by the Department of Science and Technology (DST), Government of India and the United States Department of Energy, Office of Nuclear Physics (DOE NP), USA, under Grant No. DE-FG02-92ER40713.
[1] J. D. Bjorken, K. L. Kowalski, and C. C. Taylor, 7th Les Rencontres de Physique de la Vallee d'Aoste: Results and
Perspectives in Particle Physics, Report No. SLAC-PUB-6109, (1993), pp. 507-528. 
[2] J. D. Bjorken, Acta Phys. Polon. B 28, 2773 (1997).

[3] K. Rajagopal and F. Wilczek, Nucl. Phys. B 399, 395 (1993).

[4] M. M. Aggarwal et al. (WA98 Collaboration), Phys. Lett. B 420, 169 (1998).

[5] M. M. Aggarwal et al. (WA98 Collaboration), Phys. Rev. C 64, 011901(R) (2001).

[6] T. C. Brooks et al. (MiniMax Collaboration), Phys. Rev. D 61, 032003 (2000).

[7] L. Adamczyk et al. (STAR Collaboration), Phys. Rev. C 91, 034905 (2015).

[8] J. I. Kapusta and S. M. H. Wong, Phys. Rev. Lett 86, 4251 (2001).

[9] S. Gavin and J. I. Kapusta, Phys. Rev. C 65, 054910 (2002).
[10] C. Pruneau, S. Gavin, and S. Voloshin, Phys. Rev. C 66, 044904 (2002).

[11] J. Schaffner-Bielich and J. Randrup, Phys. Rev. C 59, 3329 (1999).

[12] B. K. Nandi, G. C. Mishra, B. Mohanty, D. P. Mahapatra, and T. K. Nayak, Phys. Letts. B 449, 109 (1999).

[13] B. K. Nandi, T. K. Nayak, B. Mohanty, D. P. Mahapatra, and Y. P. Viyogi, Phys. Lett. B 461, 142 (1999).

[14] X. N. Wang and M. Gyulassy, Phys. Rev. D 44, 3501 (1991).

[15] Zi-Wei Lin, Che Ming Ko, Bao-An Li, Bin Zhang, and Subrata Pal, Phys. Rev. C 72, 064901 (2005).

[16] R. Nayak (ALICE Collaboration), MDPI Proc. 10, 22 (2019). 